\title{
Dominant decisions by argumentation agents
}

\author{
Paul-Amaury Matt, Francesca Toni, and Juan R. Vaccari \\ Department of Computing, Imperial College London, UK \\ \{pmatt,f.toni, juan.vaccari08\}@imperial.ac.uk
}

\begin{abstract}
We introduce a special family of (assumption-based argumentation) frameworks for reasoning about the benefits of decisions. These frameworks can be used for representing the knowledge of intelligent agents that can autonomously choose the "best" decisions, given subjective needs and preferences of decision-makers they "represent". We understand "best" decisions as dominant ones, giving more benefits than any other decisions. Dominant decisions correspond, within the family of argumentation frameworks considered, to admissible arguments. We also propose the use of degrees of admissibility of arguments as a heuristic to assess subjectively the value of decisions and rank them from "best" (dominant) to "worst". We extend this method to provide notion of relative value of decisions where preferences over benefits are taken into account. Finally, we show how our techniques can be successfully applied to the problem of selecting satellite images to monitor oil spills, to support electronic marketplaces for earth observation products.
\end{abstract}

\section{Introduction}

This paper presents two methods for evaluating and ranking (respectively) decisions using assumption-based argumentation (ABA) frameworks $[4,13,14]$. These ABA frameworks can be used for representing the knowledge of intelligent agents that autonomously make the best decision, e.g. by choosing the "best" items/ services available in a service-oriented architecture, e.g. as envisaged in [32]. We define a notion of dominance to characterise "best" items, and show that these items are those that correspond, within the family of argumentation frameworks considered, to admissible arguments as defined in $[4,13]$. Intuitively, the method relies upon comparing the value of different items by "arguing" about the benefits they provide. The reason why an item provides a benefit is explained logically (by means of arguments). ABA allows to study collections of logically constructed arguments based upon assumptions. As we shall see, ABA frameworks are particularly adequate for representing and reasoning about the benefits of items as well as for providing explanations to users.

In practice, decision-makers do not always choose top-quality ("best") items, but often choose items which fit their budget and have highest quality/price ratio. In this context, it is useful to assess numerically the quality or relative quality of items. We propose a novel criterion (or "semantics", as understood in argumentation) for choosing sets of assumptions and arguments. This criterion 
assigns degrees of admissibility to sets of assumptions, measuring the fraction of counter-attacked arguments against the given sets. We show that this criterion allows to rank items rather than simply classifying items as good (dominant/admissible) or bad (non-dominant/non-admissible). We also propose a generalisation of the formula for degrees of admissibility of decisions to take numerically into account the customer's preferences over benefits or goals, thus giving the relative value of decisions. Overall, we thus propose three argumentationbased methods for comparing decisions: conventional admissibility, degrees of admissibility, and relative value of decisions. In decision-theoretic terms, our work can be classified as providing three methods for solving problems of decision making under certainty.

Our methods are defined for simple decision frameworks. We believe that these frameworks have nonetheless wide applicability. In particular, they are suitable to support decision-making in electronic marketplaces. These allow internet users to meet business organisations, to discover, select and purchase their goods or services. Motivated by our involvement in the ARGUGRID project ${ }^{1}$, one of our goals and aspirations for this work is to propose tools for supporting users during the selection phase of this business process and notably, to empower the user with expert insight on specific categories of goods and services. We demonstrate the potential of our approach by dealing with the problem of selecting satellite images to monitor oil spills, to support electronic marketplaces for earth observation products.

The paper is organised as follows. We briefly introduce ABA in section 2. In section 3 we describe the kind of decision problems we consider in this paper and the notion of dominance to characterise the "best" decisions amongst a set of possible ones. In section 4 we propose a special family of ABA frameworks suitable for representing and reasoning about the benefits of decisions. We also prove that, within the family of frameworks considered, the notion of dominance is equivalent to the semantics of admissibility in argumentation. Section 5 discusses the use of degrees of admissibility as a low complexity heuristic for assessing the value of decisions and for ranking then from "best" to "worst". In section 6 , the notion of dominance and the heuristic are applied to the industrial procurement of satellite images in the case of oil spills. We discuss related work (from decision theory and argumentation-based decision making) in section 7 and finally conclude in section 8 .

\section{Background: Assumption-based argumentation}

Here we provide essential background on assumption-based argumentation (ABA), more details can be found in $[4,13,10,14]$.

An $A B A$ framework is a quadruple $\langle\mathcal{L}, \mathcal{R}, \mathcal{A}, \mathcal{C}\rangle$ where

$-\mathcal{L}$ is a set of sentences, referred to as language

\footnotetext{
$1_{\text {www .argugrid.eu }}$
} 
$-\mathcal{R}$ is a set of (inference) rules of the form $\frac{p_{1}, \ldots, p_{n}}{q}$ for $n \geq 0$, where $p_{1}, \ldots, p_{n} \in$ $\mathcal{L}$ are called the premises and $q \in \mathcal{L}$ are called the conclusion of the rule

$-\mathcal{A} \subseteq \mathcal{L}$, referred to as the set of assumptions

$-\mathcal{C}: \mathcal{A} \rightarrow \mathcal{L}$, referred to as the contrary mapping.

As in $[13,10]$, we will restrict attention to flat ABA frameworks, such that if $l \in \mathcal{A}$, then there exists no rule of the form $\frac{p_{1}, \ldots p_{n}}{l} \in \mathcal{R}$ for any $n \geq 0$.

Arguments are deductions of conclusions supported by assumptions. Arguments can be represented as trees having conclusions as their roots and assumptions as their leaves [14]. These trees can be computed in a forward or backward manner. If computed backwards, an argument $A \vdash p$ with conclusion $p \in \mathcal{L}$ and supported by the set of assumptions $A \subseteq \mathcal{A}$ is a finite sequence of multisets of sentences $S_{1}, \ldots, S_{m}$, where $S_{1}=\{p\}, S_{m}=A$, and for every $1 \leq i<m$, where $\sigma$ is the sentence occurrence in $S_{i}$ selected by a given selection function:

1. if $\sigma \notin A$ then $S_{i+1}=S_{i}-\{\sigma\} \cup S$ for some inference rule of the form $\frac{S}{\sigma} \in \mathcal{R}$

2. if $\sigma \in A$ then $S_{i+1}=S_{i}$.

This backward computation of arguments is employed within all computational tools for ABA (e.g. see [20]).

Given two sets of assumptions $A$ and $B$, we say that the set $A$ attacks $B$ if and only if there exists an argument $A^{\prime} \vdash p$ for $A^{\prime} \subseteq A$ whose conclusion is the contrary of some assumption $b \in B$, or formally when there exists $b \in B$ such that $p=\mathcal{C}(b)$. In other words, $A$ attacks $B$ if a subset of $A$ supports an argument that "contradicts" an assumption in $B$.

This notion of attack is a binary relation over sets of assumptions which can be represented by a set of pairs attacks $\subseteq \mathcal{A} \times \mathcal{A}$. Properties of sets of assumptions, aimed at sanctioning them (and the arguments they support) as "acceptable", can be defined based on such a relationship. "Acceptable" sets of assumptions represent the rational arguments that intelligent agents may use to support their opinions.

In the literature, several notions of "acceptability", referred to as extensionbased semantics, have been defined and studied $[12,4,13,10]$. In this paper we are concerned with the semantics of admissibility. Formally, given a set of assumptions $A \subseteq \mathcal{A}$

- $A$ is conflict-free if and only if $A$ does not attack itself

- $A$ is admissible if and only if $A$ is conflict-free and attacks every set $B$ that attacks $A$

The language of an ABA framework may contain sentences representing properties of interest for an agent such as goals, beliefs and possible actions or decisions $[33,11]$. The set of inference rules gives a logical structure to the language. The inference rules are used to model either known or observed facts and construct arguments to support claims. Assumptions play the role of beliefs which are not directly observable or known, but are useful in building arguments. Arguments are justified claims, but they do not have the value of proofs as they are disputable, so agents need semantics such as admissibility to elaborate rational opinions. 


\section{Dominant decisions}

We will consider simple decision-making settings where a user/agent needs to decide which element of a given (finite) set to choose. This set can be thought of as consisting of mutually exclusive items. Different items will typically bring the user/agent different benefits (or goals), according to the features of these items as well as some general beliefs of the user/agent concerning how these features affect the benefits. Formally, in this paper a decision framework is a tuple $(D, G, F, B)$ where

- $D$ is a (finite) set of (mutually exclusive) decisions

- $G$ is a (finite) set of benefits/goals that the user wishes to achieve

- $F$ is a (finite) set of features that items may exhibit

$-B$ is a (finite) set of beliefs concerning (i) the features that items actually exhibit and (ii) the achievement of goals, given (some) features

$B$ can be seen as a set of beliefs on which to base opinions regarding the value of items towards achieving benefits. We will assume that $B$ is a set of implications, respectively of the form:

$$
\begin{aligned}
& \text { (i) } d \rightarrow f \\
& \text { (ii) } f_{1}, \ldots, f_{n}, s g_{1}, \ldots, s g_{m} \rightarrow g \quad(n, m \geq 0, n+m>0)
\end{aligned}
$$

where $d \in D, f, f_{i} \in F$ (for $\left.i=1, \ldots, n\right), g, s g_{j} \in G \cup S G$ (for $j=1, \ldots, m$ ) with $S G$ a given set of sub-goals, $S G \cap G=\{\} .^{2}$ An implication $d \rightarrow f \in B$ stands for "item $d$ has feature $f$ ". An implication $f_{1}, \ldots, f_{n}, s g_{1}, \ldots, s g_{m} \rightarrow g \in B$ stands for "features $f_{1}, \ldots, f_{n}$ and the achievement of sub-goals $s g_{1}, \ldots, s g_{m}$ guarantee the achievement of (goal or sub-goal) $g$ ". The first kind of implication is specific to given available decisions, whereas the second kind is generic and represents beliefs that would hold even for different available decisions.

As we will dicuss in section 6 , although simple, our decision frameworks can be used to represent realistic, industrial applications, e.g. a wide range of eprocurement problems. Note that decision frameworks may accompany or be obtained from influence diagrams [7], that are widely used representation tools to support decision-making.

Until section 6, we will use, for illustration purposes, a toy scenario whereby a user/agent needs to choose accommodation in London that is close to Imperial College (represented as near) and reasonably priced (represented as cheap), and considers as options a hotel (referred to as $j h$ ), Imperial college student halls (referred to as $i c$ ), both in South Kensington, and the Ritz hotel (referred to as ritz), in Piccadilly. This scenario can be represented by a decision problem with

$-D=\{j h, i c, r i t z\}$

$-G=\{$ cheap, near $\}$

\footnotetext{
${ }^{2}$ Note that we do not include $S G$ explicitly as a component of the decision framework, since this can be drawn from the other components, namely $S G$ is the set of all sentences occurring in $B$ but not in $D \cup F \cup G$.
} 


$$
\begin{aligned}
& -F=\{\text { price }=50 £, \text { price }=70 £, \text { price }=200 £, \\
& \text { inSouthKen, inPiccadilly }\} \\
& \text { - } B=\{\text { ic } \rightarrow \text { price }=50 £, j h \rightarrow \text { price }=70 £, \text { rit } z \rightarrow \text { price }=200 £ \text {, } \\
& i c \rightarrow \text { inSouthKen, } j h \rightarrow \text { inSouthKen, rit } \rightarrow \text { inPiccadilly, } \\
& \text { price } \leq 50 £ \rightarrow \text { cheap, inSouthKen } \rightarrow \text { near, } \\
& \text { price }=50 £ \rightarrow \text { price } \leq 50 £ \text {, } \\
& \text { price }=70 £ \rightarrow \text { price }>50 £, \text { price }=200 £ \rightarrow \text { price }>50 £\}
\end{aligned}
$$

The set of sub-goals underlying this decision problem is $S G=\{$ price $\leq 50 £$, price $>50 £\}$.

In order to determine the "value" of an item one shall first find out which benefits the item provides.

Definition 1 ( $\gamma$-value of an item). The value of an item $d \in D$ is given by

$$
\gamma(d)=\left\{g \in G \mid B \cup\{d\} \vdash_{M P} g\right\}
$$

where $\vdash_{M P}$ stands for repeated applications of the modus ponens inference rule for $\rightarrow .^{3}$

In our toy scenario, $\gamma($ ic $)=\{$ cheap, near $\}, \gamma(j h)=\{$ near $\}$ and $\gamma($ ritz $)=\{\}$.

If the value of an item $d$ is bigger (in the sense of set inclusion) than the value of any other item, then the item $d$ basically provides all benefits that can be achieved by any other item and is undoubtedly "best". We call any such item "dominant".

Definition 2 (Dominance). An item $d \in D$ is dominant if and only if $\gamma(d) \supseteq$ $\gamma\left(d^{\prime}\right)$ for every $d^{\prime} \in D-\{d\}$.

In our toy scenario, $i c$ is a dominant item/choice, since its value includes both goals in $G$. No other item is dominant.

Note that other notions of dominance are possible, for example considering the number of benefits given by decisions. We plan to explore other such notions in the future.

\section{A family of ABA frameworks for comparing decisions}

We introduce a family of $\mathrm{ABA}$ frameworks $\langle\mathcal{L}, \mathcal{R}, \mathcal{A}, \mathcal{C}\rangle$ for comparing decisions, given a simple decision framework $(D, G, F, B)$ (with set of sub-goals $S G$ ) as outlined in section 3. For every item $d \in D$, feature $f \in F$ and benefit $g \in G$, let us introduce the following sentences in $\mathcal{L}$ :

- $f^{d}$, standing for "item $d$ has feature $f$ "

$-g^{d}$, standing for "benefit or sub-goal $g$ is provided by item $d$ "

- $M\left(\neg g^{d}\right)$, standing for "item $d$ cannot provide benefit $g$ "

\footnotetext{
${ }^{3}$ The modus ponens inference rule amounts to deriving $c$ from $a \rightarrow c$ and $a$, for any set (conjunction) of sentences $a$ and sentence $c$.
} 
$-\neg d$, standing for "the user does not choose item $d$ "

For example, the sentence near ${ }^{r i t z}$ stands for "the ritz hotel is close to Imperial College", the sentence price $=50 £^{j h}$ stands for "the $j h$ hotel costs $50 £$ per night", and the sentence $\neg i c$ stands for "the user does not choose $i c$ ".

Given these sentences, we formally define $\langle\mathcal{L}, \mathcal{R}, \mathcal{A}, \mathcal{C}\rangle$ as follows:

- the language

$$
\begin{aligned}
\mathcal{L}= & D \cup\{\neg d \mid d \in D\} \cup\left\{f^{d} \mid f \in F, d \in D\right\} \cup \\
& \left\{g^{d}, M\left(\neg g^{d}\right) \mid(g, d) \in G \cup S G \times D\right\}
\end{aligned}
$$

- the inference rules $\mathcal{R}$ consist of all the rules of the form

- $\frac{d}{f^{d}}$ if $d \rightarrow f \in B$, for $d \in D$ and $f \in F$

- $\frac{d, f_{1}^{d}, \ldots, f_{n}^{d}, s g_{1}^{d}, \ldots, s g_{m}^{d}}{S G, g^{d}}$ if $f_{1}, \ldots, f_{n}, s g_{1}, \ldots, s g_{m} \rightarrow g \in B$, for $f_{i} \in F, s g_{j} \in$ $S G, g \in S G \cup G$

- $\frac{g^{d^{\prime}}, M\left(\neg g^{d}\right)}{\neg d}$ for every benefit $g \in G$ and pair of distinct items $d, d^{\prime} \in D$

- the assumptions

$$
\mathcal{A}=D \cup\left\{M\left(\neg g^{d}\right) \mid(g, d) \in G \times D\right\}
$$

- the contrary mapping $\mathcal{C}: \mathcal{A} \rightarrow \mathcal{L}$, defined, for all $a \in \mathcal{A}$, as

$$
\mathcal{C}(a)=\left\{\begin{array}{l}
\neg d \text { if } a=d \\
g^{d} \text { if } a=M\left(\neg g^{d}\right)
\end{array}\right.
$$

The first two types of inference rules in $\mathcal{R}$ can be automatically obtained from $B$ in the given decision framework. In our toy scenario, these include

$$
\begin{aligned}
& \frac{i c}{\text { price }=50 £^{i c}} \quad \frac{j h}{\text { inSouthKen }}{ }^{j h} \\
& \frac{j h, \text { inSouthKen }}{\text { near }}{ }^{j h} \quad \frac{i c, \text { price } \leq 50 £^{i c}}{\text { cheap }^{i c}}
\end{aligned}
$$

These rules allow the generation of arguments of the form

$$
\{d\} \vdash g^{d}
$$

playing the role of expert proofs that a given item $d$ allows to achieve a benefit $g$. Examples of these arguments in our toy scenario are $\{i c\} \vdash$ cheap and $\{j h\} \vdash$ near. The third type of rules allow to express reasons for not taking decisions, motivated by other decisions fulfilling goals that the current decision can be assumed not to fulfil. Examples of these rules in our toy scenario include

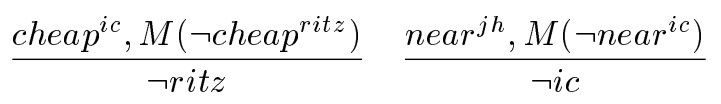


Reasons for not choosing particular items are arguments of the form

$$
\left\{d^{\prime}, M\left(\neg g^{d}\right)\right\} \vdash \neg d
$$

for any $d^{\prime}$ giving benefit $g$ (namely such that an argument $\left\{d^{\prime}\right\} \vdash g^{d^{\prime}}$ exists). In our toy scenario, these arguments include $\left\{i c, M\left(\neg\right.\right.$ cheap $\left.\left.^{\text {ritz }}\right)\right\} \vdash \neg$ ritz (since $\{i c\} \vdash$ cheap $\left.^{i c}\right)$.

The processing of all these arguments allows us to compare the value of all items in a rigorous and automated fashion. Indeed, given the ABA framework corresponding to a decision framework as detailed above, the dominant items, providing the largest number of benefits as defined in section 3 , can be characterised by the semantics of admissibility for the ABA framework, as given by theorem 1 below. In the remainder of the paper we will assume as given a decision framework $(D, G, F, B)$ and the corresponding ABA framework $\langle\mathcal{L}, \mathcal{R}, \mathcal{A}, \mathcal{C}\rangle$.

The proof of theorem 1 relies upon the following lemma:

Lemma 1. Given an item $d \in D$, there is an argument $\{d\} \vdash g^{d}$ (with respect to $\langle\mathcal{L}, \mathcal{R}, \mathcal{A}, \mathcal{C}\rangle)$ if and only if $g \in \gamma(d)$ (with respect to $(D, G, F, B)$ ).

This directly follows from the fact that $\{d\} \vdash g^{d}$ if and only if $\{d\} \vdash_{M P} g$.

Theorem 1 (Dominance \& admissibility). Given an item $d \in D,\{d\}$ is admissible (with respect to $\langle\mathcal{L}, \mathcal{R}, \mathcal{A}, \mathcal{C}\rangle$ ) if and only if $d$ is dominant (with respect to $(D, G, F, B)$ ).

Proof. $\Rightarrow$ : By contradiction, assume that $d$ is not dominant. Then, by definition of dominance, $\exists d^{\prime} \neq d$ such that $\gamma(d) \nsupseteq \gamma\left(d^{\prime}\right)$. Thus, $\exists g \in \gamma\left(d^{\prime}\right)$ such that $g \notin \gamma(d)$. By lemma 1 and by construction of the ABA framework, $\exists g \in \gamma\left(d^{\prime}\right)$ means that there exists an argument $\left\{d^{\prime}, M\left(\neg g^{d}\right)\right\} \vdash \neg d$ attacking $\{d\}^{4}$, given that $\mathcal{C}(d)=\neg d$. Since $\{d\}$ is admissible, $\{d\}$ counter-attacks $\left\{d^{\prime}, M\left(\neg g^{d}\right)\right\} .\{d\}$ does not attack $\left\{d^{\prime}\right\}$ because the only way to attack $\left\{d^{\prime}\right\}$ is by arguments with support containing $M\left(\neg g^{d^{\prime}}\right)$ and some other $d^{*}$, trivially not contained in $\{d\}$. By elimination, $\{d\}$ attacks $M\left(\neg g^{d}\right)$. Since $\mathcal{C}\left(M\left(\neg g^{d}\right)\right)=g^{d}$, by lemma 1 , there exists an argument $\{d\} \vdash g^{d}$. Therefore, again by lemma $1, g \in \gamma(d)$, which is absurd. By contradiction, $d$ is dominant.

$\Leftarrow:\{d\}$ is necessarily conflict-free since there is no argument supported solely by $\{d\}$ for $\mathcal{C}(d)=\neg d$. Let $X \vdash \neg d$ be an arbitrary argument attacking $\{d\}$. By construction of the ABA framework, there must exist a benefit $g$ such that $M\left(\neg g^{d}\right) \in X$ and a decision $d^{\prime} \in X$ such that $\left\{d^{\prime}\right\} \vdash g^{d^{\prime}}$. By lemma $1, g \in \gamma\left(d^{\prime}\right)$. By dominance of $d, g \in \gamma(d)$ too. Again by lemma $1,\{d\} \vdash g^{d}$, which attacks $M\left(\neg g^{d}\right) \in X$. Thus, $\{d\}$ counter-attacks $X$. Since $X$ was chosen arbitrarily, $\{d\}$ is an admissible set of assumptions.

\footnotetext{
${ }^{4}$ An argument attacks a set of assumption if and only if the conclusion of the argument is the contrary of an assumption in the set. If an argument attacks a set of assumptions, then the support of the argument attacks the set of assumptions too.
} 
Therefore, argumentation agents can check whether an item $d$ is the "best" or dominant by testing whether (the singleton set consisting of) this item is admissible. For our toy scenario, the only dominant item is $i c$, since $\{i c\}$ is the only admissible set consisting of a single decision. Any software able to compute admissible sets of assumptions for an ABA framework can be used to identify dominant items. In particular, the CaSAPI ABA engine [20] can be used for this purpose.

\section{Degrees of admissibility}

This section builds upon the previous theorem 1 and aims at obtaining a way of ranking items rather than simply classifying items as good (dominant/admissible) or bad (non-dominant/admissible). The idea is to assess degrees of admissibility instead of simply checking admissibility. In practice, decision-makers do not always choose items of highest quality, but more often choose those which fit their budget and have highest quality/price ratio. In this context, it becomes necessary to assess numerically the quality or relative quality of items. For example, consider a variant of our toy scenario where the beliefs about cheap in $B$ are replaced by:

$$
\begin{aligned}
& \text { price } \leq 70 £ \rightarrow \text { cheap }, \\
& \text { price }=50 £ \rightarrow \text { price } \leq 70 £, \text { price }=70 £ \rightarrow \text { price } \leq 70 £, \\
& \text { price }=200 £ \rightarrow \text { price }>70 £
\end{aligned}
$$

Then, both $\{i c\}$ and $\{j h\}$ are dominant, but $i c$ has a better quality/price ratio. If we consider one further goal luxury, and add

$$
\begin{aligned}
& \text { - to } G: \text { luxury } \\
& \text { - to } F: 4 \text { star } \\
& \text { - to } B: j h \rightarrow 4 \text { star, } 4 \text { star } \rightarrow \text { luxury }
\end{aligned}
$$

then the only dominant/admissible decision is $j h$, but $i c$ may have a better quality/price ratio still.

In order to generalise the notion of admissibility, we introduce a scale of degrees of admissibility for sets of assumptions which ranges from 0 to 1 . Admissible sets correspond to those with a degree of 1 . Sets which are not conflict-free have a degree of 0 . Sets which are conflict-free but do not counter-attack all arguments attacking them have a degree comprised between 0 and 1 .

For these sets, one can use the fraction of counter-attacked arguments as degree of admissibility. This measure is intuitively appealing, as the higher this fraction for a decision $d$, the more benefits $d$ provides.

Definition 3 ( $\alpha$-degree of admissibility). Given an $A B A$ framework $\langle\mathcal{L}, \mathcal{R}$, $\mathcal{A}, \mathcal{C}\rangle$ and $A \subseteq \mathcal{A}$, let $X=\{B \mid B$ attacks $A\}$. Then, the degree of admissibility of $A$ is given $b y^{5}$

\footnotetext{
${ }^{5}$ Here, $|S|$ stands for the cardinality of set $S$.
} 


$$
\alpha(A)=\left\{\begin{array}{l}
1 \text { if there are no attacks against } A(\text { namely } X=\{\}) \\
\left.0 \text { if } A \text { is not conflict-free (namely } A^{\prime} \in X \text { for some } A^{\prime} \subseteq A\right) \\
\frac{\mid\{B \mid B \in X \text { and } A \text { attacks } B\} \mid}{|X|} \text { otherwise }
\end{array}\right.
$$

In the revised toy scenario of this section, $\alpha(\{j h\})=1, \alpha(\{i c\})=2 / 3$ and $\alpha(\{r i t z\})=0$. Note that, in the specific decision-making setting studied in this paper, (the set of assumptions consisting of) any decision is necessarily conflictfree, and the case of $\alpha(\{d\})=0$ is "reserved" for non-dominant decisions $d$ fulfilling no goals.

Trivially, $A$ is admissible if and only if $\alpha(A)=1$. Thus, for $\langle\mathcal{L}, \mathcal{R}, \mathcal{A}, \mathcal{C}\rangle$ the ABA framework corresponding to a decision framework $(D, G, F, B)$, directly from theorem 1:

Corollary 1 (dominance \& (degrees of) admissibility). For every item $d \in D$, the following three propositions are equivalent:

$$
\begin{aligned}
& -\{d\} \text { is admissible } \\
& -d \text { is dominant } \\
& -\alpha(\{d\})=1
\end{aligned}
$$

In the case of decision frameworks, degrees of admissibility are quite simple to compute. For a given item $d \in D$, we are interested in the arguments that attack $d$ and those supported by $\{d\}$ which counter-attack them. There exist as many attacks against $d$ as there are arguments supporting sentences of the form $g^{d^{i}}$, where $d^{\prime} \in D$ is an item distinct from $d$. Let us then introduce the following notations

$$
\begin{aligned}
& -T_{d, g}= \begin{cases}1 & \text { if there exists an argument supporting } g^{d} \\
0 & \text { otherwise }\end{cases} \\
& -T_{g}=\sum_{d \in D} T_{d, g} \\
& -T_{d}=\sum_{g \in G} T_{d, g} \\
& -T=\sum_{g \in G} T_{g}
\end{aligned}
$$

Theorem 2 (Degree of admissibility of a decision). Given a decision framework $(D, G, F, D)$ and $d \in D$ :

$$
\alpha(\{d\})=\left\{\begin{array}{l}
1 \text { if } T-T_{d}=0 \\
\frac{\sum_{g \in G} T_{d, g *}\left(T_{g}-T_{d, g}\right)}{T-T_{d}} \text { otherwise }
\end{array}\right.
$$

Proof. $\{d\}$ is definitely conflict-free. The number of arguments attacking $d$ is given by the sum

$$
\sum_{g \in G} \sum_{d^{\prime} \in D-\{d\}} T_{d^{\prime}, g}=\sum_{g \in G}\left(T_{g}-T_{d, g}\right)=T-T_{d}
$$

Assume $T-T_{d} \neq 0$. The only way to counter-attack these arguments is to attack their assumption of the form $M\left(\neg g^{d}\right)$. Clearly, each attacking argument 
supported by $\left\{M\left(\neg g^{d}\right)\right\}$ is in turn counter-attacked on the condition that there exists at least one argument supported by $\{d\}$ and supporting $g^{d}$. Consequently, the number of counter-attacks is

$$
\sum_{g \in G} \sum_{d^{\prime} \in D-\{d\}} T_{d, g} * T_{d^{\prime}, g}
$$

This last expression can be slightly simplified as

$$
\sum_{g \in G} T_{d, g} *\left(T_{g}-T_{d, g}\right)
$$

Thus, by definition of $\alpha$, the theorem holds.

Assume $T-T_{d}=0$. Then, for every $g$ and every $d^{\prime} \neq d, T_{d^{\prime}, g}=0$ and $\gamma\left(d^{\prime}\right)=\{\}$, which is trivially contained in $\gamma(d)$. Thus $d$ is dominant and, by corollary $1, \alpha(\{d\})=1$ and the theorem holds.

Note that the computation of the values $T_{d, g}$ and thus of degrees of admissibility for decisions simply requires the implementation of a program able to test the existence of arguments supporting goals in an ABA framework. Such programs are understandably rather simple to develop (see the next section 6 for a discussion concerning implementation).

By definition of degree of admissibility, if an item does not provide any benefit at all, its degree is that of a non-conflict-free set, namely 0 . The more benefits can be achieved with an item, the higher its admissibility degree. Therefore, the notion of degree of admissibility can be seen as playing the role of a relative utility function to make inter-item comparisons. This role is even clearer if we consider "weights". Indeed, decision makers may attach various degrees of importance to their goals. For example, in our toy scenario, the user may think that location is more important than luxury. In ABA, attacks or counter-attacks have no strength. In practice, however, we may want that certain attacks have more impact than others because they relate to more important issues. We show how to modify the formula for degrees of admissibility so as to incorporate the importance of the customer's goals and more accurately reflect her/his true preferences. Thus, instead of assigning the same importance to all benefits in $G$, we introduce weights $w(g)>0$ for all $g \in G$, representing the importance or level of priority of benefit $g$. Note that these weights can be any and need not sum up to one. The previously established formula for the degree of admissibility as given in theorem 2 can be naturally generalised to the following

Definition 4 (Relative value of a decision). Given $d \in D$, the relative value of $d$ is given by

$$
\alpha^{*}(d)=\frac{\sum_{g \in G} w(g) * T_{d, g} *\left(T_{g}-T_{d, g}\right)}{\sum_{g \in G} w(g) *\left(T_{g}-T_{d, g}\right)}
$$

In our toy scenario, assume $w($ cheap $)=2, w($ near $)=3$ and $w($ luxury $)=1$. Then, $\alpha^{*}(j h)=5 / 6, \alpha^{*}(i c)=1$ and $\alpha^{*}($ ritz $)=0$. 
Note that, if there exists some constant $\lambda>0$ such that for all $g \in G$ the importance of the goal $g$ is $w(g)=\lambda$, then for every item $d \in D$ it is clear that $\alpha^{*}(d)=\alpha(d)$, i.e. the notion of relative value of an item collapses with the one of degree of admissibility. Thus, we may say that the notion of relative value of an item generalises in the mathematical sense the notions of admissibility and dominance of the item. $\alpha^{*}$ measures how close an item $d$ is to satisfy the property of dominance.

\section{Supporting e-marketplaces}

Electronic marketplaces allow internet users to meet business organisations, to discover, select and purchase their goods, products or services. We have implemented the methods for decision-making described in this paper (dominance $=$ admissibility and degrees of admissibility) and have tested them on an emarket place application, for the selection of earth observation products, namely images generated by satellites. Earth observation satellites are specifically designed to observe earth from orbit, and intended for environmental monitoring, meteorology, and maps making. The availability of satellites with different characteristics (type of sensors, types of orbits, etc.) and the pecularities of the images needed by users add complexity to the problem of selecting earth observation products.

The specific earth observation problem described in this section is the problem of selecting satellite images to monitor oil spills. This problem has been proposed by GMV S.A. ${ }^{6}$, building upon their extensive field experience. The full problem is described in [31]. In this section we concentrate on dealing with a fragment of this problem, in order to focus on the representation of this kind of problems in terms of a decision framework $(D, G, F, B)$ as understood in this paper. Using the general-purpose method described in section 4 , this decision framework can then be mapped onto an argumentation framework to which the methods of sections 4 and 5 can be applied.

We consider two satellites, referred to as sat 1 and sat2, available for choosing images needed to observe a given oil spill. Thus,

$$
D=\{\operatorname{sat} 1, \text { sat } 2\} \text {. }
$$

The required satellite must accomplish several goals such as timing and quality of images for the particular oil spill setting. We are going to focus on one such goal, namely (a required) radiometric resolution (radiometric_resolution). Thus,

$$
G=\{\text { radiometric_resolution }\} \text {. }
$$

We define $B$ incrementally, as the union of several sets of implications.

In order to accomplish the required radiometric resolution, the selected satellite must be able to distinguish differences of intensity in the images. The differences can be expressed in terms of color emission, temperature, radiation reflection or altitude. These can all be seen as sub-goals.

\footnotetext{
${ }^{6}$ http: //www.gmv.com
} 


$$
\begin{aligned}
B 1=\{ & \text { color_emission } \rightarrow \text { radiometric_resolution, }, \\
& \text { temperature } \rightarrow \text { radiometric_resolution, } \\
& \text { radiation_reflection } \rightarrow \text { radiometric_resolution, }, \\
& \text { altitude } \rightarrow \text { radiometric_resolution }\}
\end{aligned}
$$

To detect differences in terms of color emission the observation must be performed during the day (day); there must be no obstacles (no_obstacle) such as rain, clouds, haze, or snow; the colours of the object to be detected and the background must be distinguishable (colors_distinguishable); and the satellite must possess a panchromatic sensor (panchromatic_sensor).

$$
\begin{array}{r}
B 2=\{d a y, \text { no_obstacle }, \text { colors_distinguishable,panchromatic_sensor } \\
\rightarrow \text { color_emission }\}
\end{array}
$$

To detect differences in terms of temperature the object and the background must have different temperatures and the satellite must possess an infrared radiometer.

$B 3=\{$ temperature_distinguishable, in frared_radiometer $\rightarrow$ temperature $\}$

To detect differences in terms of radiation reflection first of all the satellite must possess a synthetic aperture radar. Then the object must reflect and the background absorbe the signal emitted by the satellite or the object must absorbe and the background reflect the signal. The object reflects the signal if it has a riddled surface and the riddles are small (up to 5 centimeters). The background reflects the signal if the background is riddled and the riddles are big (larger than 5 centimeters). The object absorbs the signal if the object surface is smooth or if the surface is riddled and the riddles are small (up to 5 centimeters). The background absorbs the signal if the background surface is smooth or if the surface is riddle and the riddles are small (up to 5 centimeters).

$$
\begin{aligned}
B 4=\{ & \text { object_riddled,object_riddles_big } \rightarrow \text { object_reflection } \\
& \text { background_riddled,background_riddles_big } \rightarrow \text { background_reflection } \\
& \text { object_smooth } \rightarrow \text { object_absorption } \\
& \text { object_riddled,object_riddles_small } \rightarrow \text { object_absorption } \\
& \text { background_smooth } \rightarrow \text { background_absorb } \\
& \text { background_riddled,background_riddles_small } \rightarrow \text { background_absorb\}}
\end{aligned}
$$

To detect differences in terms of altitude the object and background must be at significantly different altitudes.

$$
B 5=\{\text { altitude_distinguishable, radar_altimeter } \rightarrow \text { altitude }\}
$$

We also have information about the satellites: sat1 possesses an infrared radiometer and a panchromatic sensor. It also counts with a radar altimeter. 
The temporal resolution for this satellite is high. The information for sat 2 is that it has a panchromatic sensor and a medium temporal resolution.

$$
\begin{aligned}
& B 6=\left\{\text { sat } 1 \rightarrow \text { infrared }_{r} \text { adiometer },\right. \\
& \text { sat } 1 \rightarrow \text { panchromatic } \text { ensor } \text {, } \\
& \text { sat } 1 \rightarrow \text { radar_altimeter, } \\
& \text { sat } 1 \rightarrow \text { temporal }_{r} \text { esolution }_{h} \text { igh } \\
& \text { sat } 2 \rightarrow \text { panchromatic_sensor, } \\
& \text { sat } 2 \rightarrow \text { radar_altimeter, } \\
& \text { sat } 2 \rightarrow \text { temporal_resolution_medium }\}
\end{aligned}
$$

To complete the presentation of $B$, we need to provide information about the environment and the characteristics of the image the user is interested in. In the specific case considered, the images must be obtained during the day, there are no obstacles in the sky, the object to capture is big and has a smooth surface and the background is riddled and those riddles are big.

$$
\begin{aligned}
B 7=\{ & \rightarrow \text { day }, \rightarrow \text { no_obstacle }, \\
& \rightarrow \text { object_smooth } \rightarrow \text { background_riddled, } \\
& \rightarrow \text { background_riddles_big, } \rightarrow \text { object_big }\}
\end{aligned}
$$

The set $B$ for the decision framework is then defined as: ${ }^{7}$

$$
B=B 1 \cup B 2 \cup B 3 \cup B 4 \cup B 5 \cup B 6 \cup B 7 .
$$

The set of features for this decision framework is

$$
\begin{aligned}
F=\{ & \text { panchromatic_sensor }, \text { in frared_radiometer,radar_altimeter }, \\
& \text { temporal_resolution_high, }, \text { temporal_resolution_medium }\} .
\end{aligned}
$$

This decision framework can be directly translated onto the corresponding ABA framework. As an example, this would include rules:

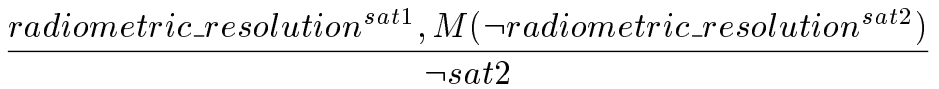

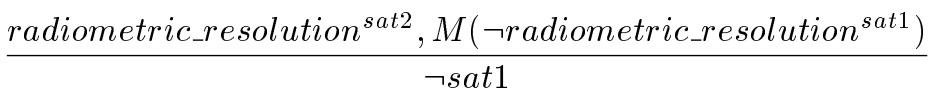

\footnotetext{
${ }^{7}$ Note that the information in $B 7$ is not strictly speaking in the format of implication beliefs as given in section 3 . We could pre-compile $B 7$ into the rest of $B$, unfolding them away from implications. We have chosen not to here because decision frameworks (and the correwsponding ABA frameworks) can be easily extended to include this "information facts" without altering any of the results in the paper.
} 
We have encoded the ABA framework as an input to CaSAPI [20] ${ }^{8}$ to obtain admissible (and thus dominant) decisions. For the simple fragment considered here, this dominant item is sat1, appropriately computed by CaSAPI.

We have also tried the the degrees of admissibility method using a Rubyon-Rails web-service prototype ${ }^{9}$. The results showed a degree of admissibility of 1.0 for sat 1 and a degree of admissibility of 0 for sat 2 . The Ruby-on-Rails web-service prototype consists of a core Ruby engine able to construct backward arguments and compute degrees of admissibility, embedded into Rails allowing to use the server via a web interface.

We have also implemented solutions to the full oil spill problem, with 5 satellites (ERS, RADARSAT, SPOT, IKONOS, METOP), 27 implications (for reasoning about images), 57 implications in total for also describing the equipment on the satellites and the scenario of exploitation, and 3 goals (radiometric, spatial and temporal image resolution). The full ABA and Ruby-on-Rails formulations can be downloaded from

http://homepage.mac.com/paulmatt/homepage/eBusiness/page3.html.

\section{Related work}

The present work can be classified in decision-theoretic terms $[15,34,19]$ as a problem of decision making under certainty. Indeed, the beliefs involved in our problem correspond to features and are by consequence controllable unknowns (secondary decisions). In this problem, argumentation is used for reasoning about the relative value of different items. In decision-theoretic terms, argumentation is used as a model to compute a utility function which is too complex to be given a simple analytical expression in closed form. Note that, broadly speaking, such limitations of classical Decision Theory have lead some authors to develop a number of qualitative approaches to Decision Theory, such as e.g. $[27,5,9]$. In this paper, we focus on using argumentation as a form of qualitative Decision Theory.

Argumentation has already been proposed for decision making, under strict uncertainty (no probabilistic information is available to the decision maker) [17, $18,1,2]$ and also for decision under risk (some probabilistic information is known) $[28,21,26,3]$. Argumentation has also been used to support practical reasoning $[29,30]$ and decision support systems $[16,24,25]$. Differently from all these approaches, in this paper we have focused on using standard Dung-style argumentation [12] as instantiated in ABA and showing the suitability of an existing semantics (admissibility) for argumentation (which fundamentally plays the same role as a decision criterion in Decision Theory) in a practical application: our theorem 1 guarantees the suitability of the notion of admissibility for studying

\footnotetext{
${ }^{8}$ CaSAPI can be downloaded from http://www.doc.ic.ac.uk/ dg00/casapi.html. The CaSAPI file for the application described in this section can be found at http://homepage . mac.com/paulmatt/homepage/eBusiness/page3.html.

${ }^{9}$ This is available at http://homepage.mac.com/paulmatt/homepage/eBusiness/page3.html
} 
choice problems in (simple, but realistic) decision frameworks. [11] also links a semantics for argumentation to a decision-theoretic criterion (Wald's minimax criterion) in a context of decision making under strict uncertainty.

The idea of using degrees of admissibility finds its origins in the notion of graduality [6]. Graduality has been introduced to generalise the classical notion of acceptability in argumentation from [12]. Degrees of acceptability have been recently used to play the role of measure of argument strength [23] and to be mathematically connected to the value of special two-person zero-sum games of strategy with imperfect information [34]. In that work however, the strength of an argument does not simply correspond to the admissibility of an extension embracing that argument, but rather the expected value of the admissibility an extension randomly chosen by a proponent of that argument, relative to the one chosen randomly by an opponent.

Some argumentation-based approaches (to decision-making as well as other applications) relies upon inducing preferences amongst sets of arguments from preferences on individual arguments (e.g. see [8]). Our computation of degrees of admissibility can be seen as a means to compute preferences amongst arguments "from first principle", solely based upon the arguments capability to counterattack (as many as possible) attacking arguments.

\section{Conclusion}

Electronic marketplaces are not only changing the way items (goods, products and services) are being offered and made available, they are also rendering the task of comparing the value of items more complex than it used to be. The need for intelligent and expert knowledge-based agents supporting both sellers and buyers in this new economy is growing fast. This paper has proposed argumentation-based agent architecture for addressing the problem of comparing items. We have emphasised the suitability of assumption-based argumentation and introduced a family of assumption-based argumentation frameworks for analysing decision problems. In these problems, decisions represent items. These frameworks can be directly deployed by (suitable) agents within an emarketplace setting.

We have proved theoretically that within this family of frameworks, the semantics of admissibility is equivalent to the concept of dominance for items. Building upon this theoretical result, we have proposed degrees of admissibility as an intuitive heuristic for comparing the value of customisable items. The original formula for the degree of admissibility of an item has then been generalised so as to take numerically into account the customer's preferences over benefits or goals. The formula obtained allows to rank subjectively the value customisable items from best to worst.

Our techniques are supported at the implementation level by two systems: the general-purpose ABA system CaSAPI and a decision-making specific Rubyon-Rails web service prototype. 
We have illustrated an application of our techniques (and systems) to the procurement of earth observation products. These techniques have also been applied to an industrial procurement problem in support of companies in the complex task of purchasing electronic ordering systems [22]. Both applications require feeding an argumentation agent with expert knowledge used to support the choice of (earth observation or e-ordering) products. The main difference between our methods and the methods currently used by industrial experts in the application areas considered lies in the amount of effort the buyer needs to put in the task of specifying the inputs to the problem. With the industrial methods, the decision-maker needs to specify the decision framework and assess the overall importance of each individual feature and grade their benefits numerically. In our approach, the decision-maker only needs to specify the decision framework (if the third method, computing the relative value, is used, the decision-maker also needs to specify numerically its preferences over the ultimate goals that can be achieved, but these are few and easy to assess). Consequently, we believe that our approach empowers the decision-maker with expert knowledge, allowing to concentrate energy on the specification and weighting of goals. In this context, the use of ABA is beneficial, with respect to the use of abstract argumentation, in that it affords a finer level of granularity in the representation.

Our preliminary experimentation with e-procurement is encouraging. We plan to further this experimentation to provide a more thorough evaluation, e.g. to assess scalability of our approach.

The use of argumentation to determine dominance allows users to be presented with explanatory information as to the reasons of the dominance (in comparison with other items concerning the relative benefits). We believe that this is an important feature of our approach when agents attempt to persuade one another (for example, if the seller of an item is told that its item is dominated by some other, then it may decide to change the specification of its item in order to persuade the buyer to change its mind). We plan to investigate this direction in the future.

We have focused on problems of decision making under certainty. Argumentation could be fruitfully used also to deal with uncertainty, e.g. as in $[17,18,1,2]$. We plan to study a suitable extension of our approach to cope with uncertainty in he future.

\section{Acknowledgements}

This work was funded by the Sixth Framework IST programme of the EC, under the 035200 ARGUGRID project. The authors would like to thank Jose Barba for useful discussions on the earth observation scenario.

\section{References}

1. L. Amgoud. A unified setting for inference and decision: An argumentation-based approach. In 21st Conference on Uncertainty in Artificial Intelligence (UAI'2005), pages $26-33,2005$. 
2. L. Amgoud and H. Prade. Making decisions from weighted arguments. In Decision theory and multi-agent planning, pages 1-14. Springer, 2006.

3. Leila Amgoud and Henri Prade. Using arguments for making decisions: A possibilistic logic approach. In 20th Conference of Uncertainty in Artificial Intelligence (UAI'04), pages 10-17, 2004.

4. A. Bondarenko, Phan Minh Dung, Robert A. Kowalski, and Francesca Toni. An abstract, argumentation-theoretic approach to default reasoning. Artificial Intelligence, 93(1-2):63-101, 1997.

5. Blai Bonet and Hector Geffner. Arguing for decisions: A qualitative model of decision making. In Proceedings of the 12th Conference on Uncertainty in Artificial Intelligence (UAI-96), pages 98-105, 1996.

6. Claudette Cayrol and Marie-Christine Lagasquie-Schiex. Graduality in argumentation. J. Artif. Intell. Res. (JAIR), 23:245-297, 2005.

7. R. T. Clemen and T. Reilly. Making Hard Decisions with Decision Tools Suite. Duxbury Resource Center, 2004.

8. Yannis Dimopoulos, Pavlos Moraitis, and Leila Amgoud. Theoretical and computational properties of preference-based argumentation. In ECAI, pages 463-467, 2008.

9. D. Dubois, H. Fargier, and P. Perny. Qualitative decision theory with preference relations and comparative uncertainty: An axiomatic approach. Artificial Intelligence, 148:219-260, 2003.

10. Phan Minh Dung, Paolo Mancarella, and Francesca Toni. Computing ideal sceptical argumentation. Artificial Intelligence, 171(10-15):642-674, 2007.

11. Phan Minh Dung, Phan Minh Thang, and Francesca Toni. Towards argumentationbased contract negotiation. In 2nd International Conference on Computational Models of Argument (COMMA'08). IOS Press, 2008.

12. P.M. Dung. On the acceptability of arguments and its fundamental role in nonmonotonic reasoning, logic programming, and n-person games. Artificial Intelligence, 77(2):321-257, 1995.

13. P.M. Dung, R.A. Kowalski, and F. Toni. Dialectic proof procedures for assumptionbased, admissible argumentation. Artificial Intelligence, 170(2):114-159, 2006.

14. P.M. Dung, R.A. Kowalski, and F. Toni. Assumption-based argumentation. In Iyad Rahwan and Guillermo Simari, editors, Argumentation in AI: The Book. SpringerVerlag, 2009. To appear.

15. Peter C. Fishburn. Decision and Value Theory. John Wiley and Sons, Inc., New York, 1964.

16. J. Fox, N. Johns, C. Lyons, A. Rahmanzadeh, R. Thomson, and P. Wilson. PROforma: a general technology for clinical decision support systems. Computer Methods and Programs in Biomedicine, 54(10-15):59-67, 1997.

17. John Fox, Paul Krause, and Morten Elvang-Gøransson. Argumentation as a general framework for uncertain reasoning. In David Heckerman and Abe Mamdani, editors, Proceedings of the 9th Conference on Uncertainty in Artificial Intelligence, pages 428-434. Morgan Kaufmann Publishers, 1993.

18. John Fox and Simon Parsons. On using arguments for reasoning about actions and values. In Jon Doyle and Richmond H. Thomason, editors, Working Papers of the AAAI Spring Symposium on Qualitative Preferences in Deliberation and Practical Reasoning, pages 55-63, 1997.

19. Simon French. Decision theory: an introduction to the mathematics of rationality. Ellis Horwood, 1987. 
20. Dorian Gaertner and Francesca Toni. Hybrid argumentation and its properties. In Antony Hunter, editor, Proceedings of the Second International Conference on Computational Models of Argument (COMMA'08). IOS Press, 2008.

21. P. Krause, S. Ambler, M. Elvang-Gøransson, and J. Fox. A logic of argumentation for reasoning under uncertainty. Computational Intelligence, 11:113-131, 1995.

22. P.-A. Matt, F. Toni, T. Stournaras, and D. Dimitrelos. Argumentation-based agents for e-procurement. In 7th International Conference on Autonomous Agents and Multiagent Systems, 2008.

23. Paul-Amaury Matt and Francesca Toni. A game-theoretic measure of argument strength for abstract argumentation. In 11th European Conference on Logics in Artificial Intelligence (JELIA 2008), 2008.

24. Sanjay Modgil and Peter Hammond. Decision support tools for clinical trial design. Artificial Intelligence in Medicine, 27(2):181-200, 2003.

25. M. Morge and P. Mancarella. The hedgehog and the fox. An argumentationbased decision support system. In Proceedings 4 th International Workshop on Argumentation in Multi-Agent Systems, 2007.

26. Simon Parsons. Normative argumentation and qualitative probability. In Dov M. Gabbay, Rudolf Kruse, Andreas Nonnengart, and Hans Jürgen Ohlbach, editors, Proceedings of the First International Joint Conference on Qualitative and Quantitative Practical Reasoning, volume 1244 of LNAI, pages 466-480. Springer, June 9121997.

27. J. Pearl. From conditional oughts to qualitative decision theory. In 9th Conference on Uncertainty in Artificial Intelligence (UAI'93, pages 12-20. 1993.

28. D. Poole. Probabilistic horn abduction and bayesian networks. Artificial Intelligence, 64(1):81-129, 1993.

29. H. Prakken. Combining sceptical epistemic reasoning with credulous practical reasoning. IOS Press, 2003.

30. Iyad Rahwan and Leila Amgoud. An argumentation-based approach for practical reasoning. In International Joint Conference on Autonomous Agents and Multiagent Systems (AAMAS), pages 347-354, 2006.

31. T. Stournaras, editor. E-business application scenario - [ARGUGRID] Deliverable D.1.2. 2007.

32. F. Toni. Argumentative kgp agents for service composition. In Proc. AITA08, Architectures for Intelligent Theory-Based Agents, AAAI Spring Symposium. Stanford University, 2008.

33. Francesca Toni. Assumption-based argumentation for selection and composition of services. In F. Sadri and K. Satoh, editors, 8th International Workshop on Computational Logic in Multi-Agent Systems (CLIMA VIII), 2007.

34. John von Neumann and Oskar Morgenstern. Theory of Games and Economic Behavior. Princeton University Press, 1944. 